\title{
Primary malignant lymphoma of the glans penis: a rare case report and review of the literature
}

\author{
Li Chu $^{1 *}$, Wei Mao ${ }^{1 *}$, Kim Curran Vikramsingh $^{1}$, Xi Liu $^{1}$, Hui-Min Qiu ${ }^{1}$, Jun-Hua Zheng ${ }^{2}$, Yi Wang ${ }^{3}$, Gen-Pei Yu ${ }^{1}$ \\ and Qing $\mathrm{Xu}^{1}$
}

Asian Journal of Andrology (2013) 15, 571-572; doi:10.1038/aja.2013.21; published online 6 May 2013

Dear Editor,

We present here a rare case of primary malignant lymphoma of the glans penis that was detected in a 72-year-old man. Additionally, we have reviewed the literature for reports of primary malignant lymphoma of the penis. This is most likely the forty-ninth case of primary malignant lymphoma of the penis reported in the medical literature.

Lymphomas typically originate from the lymph nodes and lymph tissues; however, the tonsils, spleen and bone marrow are also vulnerable sites. Due to the presence of lymph nodes and lymph tissue throughout the human body, primary malignant lymphomas of glans penis occur rarely. The diagnosis of this rare lymphoma may be difficult and delayed without specific symptoms, and treatment modalities are controversial because of the limited number of patients.

A 72-year-old male patient was referred to our hospital due to a mass on the left side of the glans penis in May 2007. A physical examination identified two masses, one on the left tip and one on the right tip of his penis. The left lump measured approximately $8 \mathrm{~mm}$ in diameter, was hard in nature, and was cystic, reddish in colour and covered the surface of the glans penis with ill-defined margins. There was no visible ulceration and no tenderness on palpation. The cystic mass on the right side measured $15 \mathrm{~mm}$; the lesion was firm in nature, did not protrude from the surface and had no palpable tenderness. The liver and spleen were not palpable, and no other sites of lymphadenopathy were observed; B symptoms were absent. Anti-inflammatory treatment was administered at another hospital, but it was unsuccessful. On 17 May 2007, the two masses were resected with penile preservation. The pathological examination showed the presence of a malignant lymphoma originating from B cells (diffuse large B), as shown in Figure 1. The patient was diagnosed with diffuse large B-cell lymphoma of the glans penis, stage IE, according to the Ann Arbor classification. No obvious distal metastasis was found by chest X-ray or abdominal ultrasonography before the surgery.

Four cycles of systemic chemotherapy with a monthly CHOP (cyclophosphamide, doxorubicin, vincristine, and prednisone) regimen were administered from June 2007 to September 2007. A follow-up of the patient in September 2007, including a chest X-ray, abdominal CT and pelvic CT, showed no obvious abnormalities.
Immunotherapy with interferon $\alpha-2 b$ was then administered until November 2007 . The patient was asymptomatic and in good general condition with no obvious distant metastasis.

In November 2007, his lactate dehydrogenase (LDH) level was $1133 \mathrm{U}^{-1}$. On 12 December 2007, PET/CT revealed a treated glans penis carcinoma. Bone marrow 18F-fluorodeoxyglucose showed slightly increased metabolism, and after clinical correlation with the patient's history, it was diagnosed as post-chemotherapy reactive hyperplasia. Moreover, whole-body PET showed no abnormal metabolic increase in the 18F-fluorodeoxyglucose uptake focus.

At a later date in December 2007, the patient started having night sweats and a dry cough with no apparent fever (B symptoms). His LDH level on 22 December 2007 was $1347 \mathrm{U}^{-1}$. From 7 January 2008 to 14 March 2008, four cycles of systemic chemotherapy with RCHOP were administered. In March 2008, his LDH was $457 \mathrm{U} \mathrm{l}^{-1}$, and his health status had improved with decreased symptoms. In December 2008, the patient developed fever with an LDH level of $783 \mathrm{U} \mathrm{l}^{-1}$. The patient presented with transient obnubilation, left limb hypodynamia, mild headache, nausea and vomiting. Beginning 1 January 2009, prednisolone and rituximab were administered intravenously. The patient's fever and appetite improved, but he subsequently went into a comatose state. On 21 February 2009, Magnatic Resonance Imaging (MRI) showed many space-occupying lesions with different shapes and sizes in the bilateral hemispheres of the brain. On the right side, larger and more numerous lesions were observed with low signal intensity in T1-weighted imaging and diffusion-weighted imaging and high signal intensity in fluidattenuated inversion recovery images with obvious oedema. The lesions were significantly strengthened after enhancement, although the area of oedema was not (Figure 2). On 22 February 2009, the patient died as a result of the ineffectiveness of the above treatment.

The first case of primary malignant lymphoma of the penis was reported in 1962. ${ }^{1}$ To the best of our knowledge, only 48 cases of primary malignant penile lymphoma have been reported in the medical literature (Supplementary Table 1). The mean reported patient age was 51.6 years (range: 4-91 years). Only two patients were younger than 18 years of age, and 11 patients were younger than 60 years of age.

${ }^{1}$ Department of Medical Oncology, Shanghai Tenth People's Hospital, Tongji University, School of Medicine, Shanghai 200072, China; ${ }^{2}$ Department of Urology, Shanghai Tenth People's Hospital, Tongii University, School of Medicine, Shanghai 200072, China and ${ }^{3}$ Department of Pathology, Shanghai Tenth People's Hospital, Tongii University, School of Medicine, Shanghai 200072, China

* These authors contributed equally to this work.

Correspondence: Dr Q Xu (xuqingmd@yahoo.com.cn)

Received: 19 November 2012; Revised: 16 December 2012; Accepted: 20 January 2013; Published online: 6 May 2013 


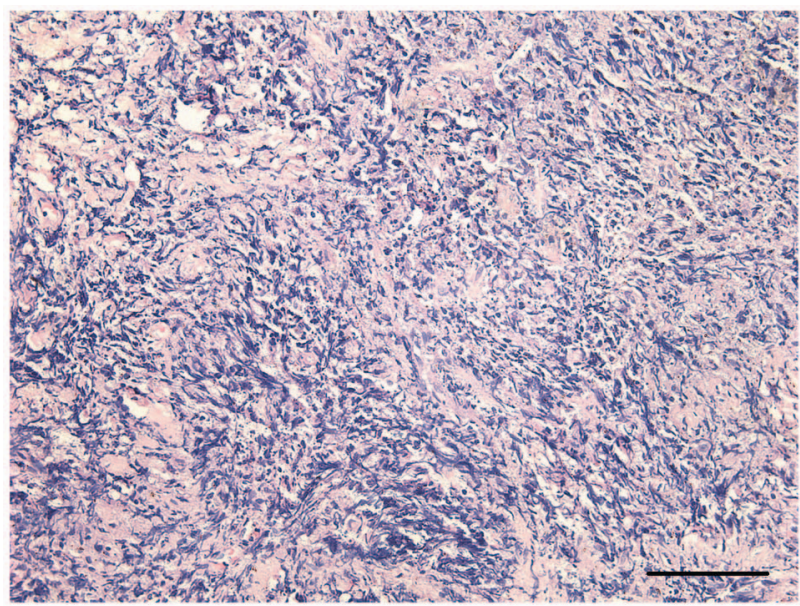

Figure 1 Diffuse penile cellular infiltration with atypical lymphoid large-size cells with polymorphic nuclei. Scale bar $=200 \mu \mathrm{m}$.

Of the 48 cases, pathological examinations were reported for only 38 cases. The pathological results showed that diffuse large B-cell lymphoma (DLBCL) was the most frequent subtype $(14 / 38){ }^{2}$ the other reported types included 10 cases of T-cell lymphoma (TCL), one case of lymphoblastic lymphoma in 2001 and one case of primitive T cellrich B-cell lymphoma of the glans penis reported in $2003 .{ }^{3} \mathrm{ALK}^{+}$ anaplastic large cell lymphoma (ALCL) of the penis was first reported in 1997. ${ }^{4}$ Additionally, one patient with extranodal marginal B-cell lymphoma of the glans penis reported in 2004 had a good prognosis. ${ }^{5}$ Two cases with mixed small and large type lymphoma have been reported, and one primary reticulum cell sarcoma type was reported in $1962 .{ }^{1}$ Metastasis has been reported in the liver and lung, among other locations, but there have been few reports of brain metastases. The most common complaint was a mass or nodule in the penis (15/ 32). Three patients complained primarily of priapism, although one complained of erectile dysfunction. A mass or nodule on the penis and organ dysfunction should be closely monitored and evaluated.

In total, 23 of 33 cases from the literature had a reported prognosis of 6 months to 4 years without recurrence and dissemination or complete remission. In our case, the disease-free survival was 21 months, and the overall survival was 25 months. Non-Hodgkin's lymphoma is more common than Hodgkin's lymphoma in these cases, and the exact subtype and stage are the main determinants of the outcome. Determining whether the prognosis for this particular carcinoma was in agreement with the current published National Comprehensive Cancer Network (NCCN) malignant lymphoma prognostic factors (e.g. age, lactate dehydrogenase level, tumour stage, Performance Status (PS) score and extranodal invasion) will require additional clinical data for verification.

Currently, there is no standard treatment for malignant lymphoma of the penis; 15 of the 42 reported cases underwent surgery, and 3 of 15 were treated solely with surgery. Additionally, 33 of the 42 reported cases were treated with chemotherapy or immunotherapy, and 9 of the 41 underwent radiotherapy. In addition to radical surgery, many cases showed that penile preservation strategies were also effective treatments for the patients. Delicato et al. ${ }^{6}$ suggested that conservative therapy could be considered as the first-choice approach with a curative goal because of the aesthetic problems and erectile dysfunction caused by surgery. Combined-modality treatments were used in some cases. Rituximab-cyclophosphamide, doxorubicin, vincristine, and prednisone, was the most common choice of systemic chemotherapy. The absence of lymphoid tissue in the penis suggests that penile
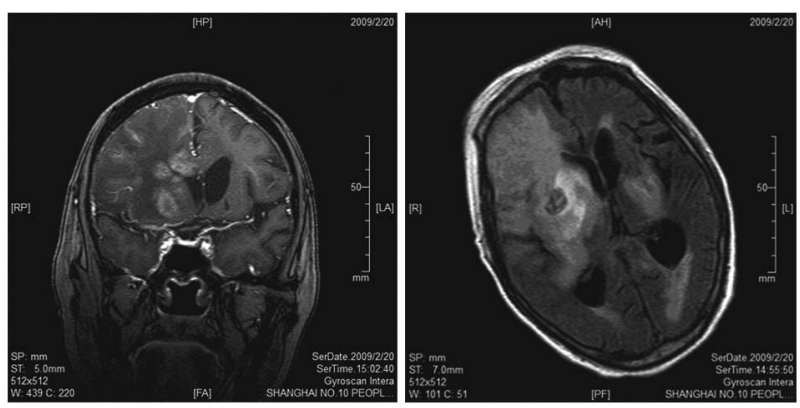

Figure 2 The MRI shows many space occupying lesions with different shapes and sizes in bilateral hemispheres of the brain. On the right side, there are more and bigger ones which are low signal in T1WI, DWI and high signal in FLAIR with obvious edema. These lesions are significant strengthened after enhancing, while the area of edema does not. DWI, diffusion-weighted imaging; FLAIR, fluid-attenuated inversion recovery; MRI, Magnatic Resonance Imaging; T1WI, T1-weighted imaging.

lymphoma is a manifestation of occult nodal disease or part of a systemic process, and this is the rationale for combined treatment modalities. ${ }^{7}$ However, patients sometimes undergo surgery that could have been avoided if the diagnosis had been considered. ${ }^{8}$

Similar relevant reports are rare, but in recent years, there have been a growing number of reports, which should make clinicians more aware of the poorly understood circumstances for the specific aetiology and pathogenesis of this disease and how to avoid misdiagnosis. Thus, early recognition of this disease and its clinical management will be maximised. Identifying a standard treatment for such patients still requires support from further clinical studies and more detailed information.

\section{AUTHOR CONTRIBUTIONS}

LC participated in the design of the study, drafted the manuscript and performed the literature review. WM participated in the study design, drafted the manuscript and performed the literature review. KCV participated in the study design and helped draft the manuscript. XL helped draft the manuscript. HMQ, JHZ, YW and GPY participated in the study design. QX conceived of the study and participated in its design and coordination and helped draft the manuscript. All authors read and approved the final manuscript.

\section{COMPETING FINANCIAL INTERESTS}

The authors declare no competing financial interests.

\section{ACKNOWLEDGMENTS}

This Article is supported by the National Natual Science Foundation of China (No. 30872591) and Shanghai Science and Technology Commission (No. 02.04.11.006).

Supplementary Information accompanies the paper on Asian Journal of Andrology website (http://www.nature.com/aja).

1 Oomura J, Ookita K, Takenaka M, Yamada S. Primary reticulosarcoma of the penis report of a case. Hinyokika Kiyo 1962; 8: 536-42.

2 Gallardo F, Pujol RM, Barranco C, Salar A. Progressive painless swelling of glans penis uncommon clinical manifestation of systemic non Hodgkin's lymphoma. Urology 2009; 73: 929.e3-5.

3 Pomara G, Cuttano MG, Tripodo C, Carlino F, Selli C. Primary T cell rich B cell lymphoma of the penis a first case. BJU Int 2003; 91: 889.

4 Cribier B, Lipsker D, Grosshans E, Duhem C, Capesius C et al. Genital ulceration revealing a primary cutaneous anaplastic lymphoma. Genitourin Med 1997; 73: 325.

5 Sadaf H, Jillian N, Ander W, Christopher W, David C. MALT lymphoma of the foreskin. Leuk Lymphoma 2004; 45: 1699-701.

6 Delicato G, Baffigo G, Bianchi D, Farullo G, Signore S et al. Conservative therapy in two cases of non-Hodgkin lymphoma of the penis: case report with review of the literature. World J Oncol 2012; 3: 37-8.

7 Arena F, Di Stefano C, Peracchia G, Barbieri A, Cortellini P. Primary lymphoma of the penis: diagnosis and treatment. Eur Urol 2001; 39: 232-5.

8 Aynsley E, Lewis J. Primary penile lymphoma: treatment with radiotherapy. Br J Med Surg Urol 2009; 2: 129-30. 